\title{
Higher order intercommutations in Cosmic String Collisions
}

\author{
A. Achúcarro* \\ Instituut-Lorentz for Theoretical Physics, Leiden, The Netherlands and \\ Departamento de Fí sica Teórica, Universidad del País Vasco UPV-EHU, Bilbao, Spain
}

G.J. Verbiest田

Instituut-Lorentz for Theoretical Physics, Leiden, The Netherlands

(Dated: October 25, 2018)

\begin{abstract}
We report the first observation of multiple intercommutation (more than two successive reconnections) of cosmic strings at ultra-high collision speeds, and the formation of "kink trains" with up to four closely spaced left- or right-moving kinks. We performed a flat space numerical study of abelian Higgs cosmic string intercommutation in the type-II regime $\beta>1$ (where $\beta=m_{\text {scalar }}^{2} / m_{\text {gauge }}^{2}$ ) up to $\beta=64$, the highest value investigated to date. Our results confirm earlier claims that the minimum critical speed for double reconnection goes down with increasing $\beta$, from $\sim 0.98 c$ at $\beta=1$ to $\sim 0.86 c$ for $\beta=64$. Furthermore, we observe a qualitative change in the process leading to the second intercommutation: if $\beta \geq 16$ it is mediated by a loop expanding from the collision point whereas if $1<\beta \leq 8$ the previously reported "loop" is just an expanding blob of radiation which has no topological features and is absorbed by the strings. The multiple reconnections are observed in the loop-mediated, deep type-II regime $\beta \geq 16$. Triple reconnections appear to be quite generic for collision parameters on the boundary between single and double reconnection. For $\beta=16$ we observe quadruple events. They result in clustering of small scale structure in the form of "kink trains". Our findings suggest that, due to the core interactions, the small scale structure and stochastic gravitational wave background of abelian Higgs strings in the strongly type-II regime may be quite different from what would be expected from studies of Nambu-Goto strings or of abelian Higgs strings in the $\beta \approx 1$ regime.
\end{abstract}

Formation of cosmic strings and superstrings is a generic outcome of cosmological phase transitions [1] and some inflationary models, both within Grand Unified theories 2, 3] and from branes [4, 5]. There is no evidence of their existence yet, but strings can lead to a wealth of astrophysical phenomena. There is an increasing number of surveys and searches looking for observable signatures such as CMB anisotropies, gravitational lensing, gravitational radiation, cosmic rays and gamma ray bursts (see classic reviews [6, 7] and more recent updates [8, 10]).

A string network is expected to reach a scaling solution in which statistical properties such as the distance between strings become a fixed fraction of the horizon size $t$. The expansion of the Universe pumps energy into the string network -by increasing the contribution of long strings- but this is balanced by energy losses to radiation. If these are efficient enough, the contribution from the strings to the energy density of the Universe remains a small, constant fraction of the dominant form of energy (matter or radiation) and is potentially observable. Radiation is emitted by oscillating loops, formed when a string self-intersects and reconnects, and in bursts. The latter are produced by cusps (sections of the string which acquire near-luminal speeds) and, to a lesser extent, by kinks created when strings reconnect. The reconnection, or intercommutation of strings is therefore an essential process that determines and maintains the long-term scaling behaviour of the string network.

Cosmic string intercommutation was first investigated numerically in Shellard's pioneering work on global strings [1] and later by Matzner 12] for type-II abelian Higgs strings with $\beta=2$ (using the type-I/type-II termi- nology from superconductors, to indicate $\beta<1 / \beta>1$ respectively). They pointed out that in ultra-relativistic collisions there is a critical center-of-mass velocity $v_{c}$ (collision angle dependent) beyond which strings pass through: a loop forms, expanding rapidly from the collision point, that catches up with the reconnected strings and produces a second intercommutation. A more recent study 13 focussed on double reconnection, which proceeds differently in type-I and type-II strings, with the loop only forming in type-II collisions. The angular dependence of $v_{c}$ is calculable from the geometry and speeds of the colliding strings 13 .

In the abelian Higgs model, all evidence to date [12, 13 ] indicates that Abrikosov-Nielsen-Olesen (ANO) strings with unit winding always reconnect at least once, even in ultra-high-speed collisions, with the possible exception of near-parallel collisions at very low speed in the typeI regime, where the attractive force between the strings can make them stick together 14. Theoretical predictions for junction formation based on the Nambu-Goto approximation 15] give an extremely good fit to numerical simulations [16].

The simulations in 13 had decreasing resolution with increasing $\beta$, and only explored two values in the type-II regime $(\beta=8,32)$, but the results suggested that the critical velocity for double reconnection would go down as a function of $\beta$. This is interesting because one of the distinguishing features of cosmic superstrings, as opposed to ANO strings, is their low intercommutation probability $P \sim 10^{-3}-10^{-1}$ [17, which leads to different scaling properties, in particular to denser networks (although the effect may be weaker than the $\rho t^{2} \sim 1 / P$ one might 
expect[18]). However, if the critical velocity of strongly type-II ANO strings decreases and becomes comparable to the average velocity of the network, the effective intercommutation probability could be much less than one.

While investigating the critical velocity for large $\beta$ we discovered higher order intercommutation events, that is, particular collisions in which the strings exchange ends more than twice. These we describe now.

\section{Simulations}

The abelian Higgs model -the relativistic GinzburgLandau model- involves a complex scalar field $\phi$ and a $\mathrm{U}(1)$ gauge field $A_{\mu}$ with field strength $F_{\mu \nu}=\partial_{\mu} A_{\nu}$ $\partial_{\nu} A_{\mu}(\mu, \nu=0,1,2,3)$. In natural units $\hbar=c=1$ it is described by the lagrangian

$\mathcal{L}=\left(\partial_{\mu}+i e A_{\mu}\right) \phi\left(\partial^{\mu}-i e A^{\mu}\right) \phi^{\dagger}-\frac{1}{4} F^{\mu \nu} F_{\mu \nu}-\frac{\lambda}{4}\left(|\phi|^{2}-\eta^{2}\right)^{2}$

The ground state has $|\phi|=\eta$ and zero magnetic field. There are two mass scales: scalar excitations have $m_{\text {scalar }}=\sqrt{\lambda} \eta$ and gauge field excitations, $m_{\text {gauge }}=$ $\sqrt{2} e \eta$. Classically, the only relevant parameter in the dynamics is their ratio, $\beta=\left(m_{\text {scalar }} / m_{\text {gauge }}\right)^{2}=\lambda / 2 e^{2}$, which also characterizes the internal structure of the ANO vortices [19, 20. In this paper we are interested in the $\beta>1$ regime, analogous to a type-II superconductor, and in this case the vortices have an inner "scalar" core of radius $\sim m_{\text {scalar }}^{-1}$ in which the scalar field departs from its vacuum value and vanishes at the center. This is surrounded by a larger, "gauge" core of radius $\sim m_{\text {gauge }}^{-1}$ where the magnetic field is non-zero. As a result, the interaction between parallel type-II strings is repulsive.

We follow the same numerical strategy as in previous studies [12, 13]: we use a lattice discretization and place a superposition of two oppositely moving ANO strings on a three dimensional lattice. This configuration is evolved using a leapfrog algorithm. The initial configuration is determined by two parameters: the center-of-mass speed $v$ of the strings when they are far apart and the angle $\alpha$ between them (every collision can be brought to this form by an appropriate Lorentz transformation [11]). We also impose "freely moving" boundary conditions: after each round the fields inside the box are updated using the equations of motion, and the fields on the boundaries are calculated assuming the strings move unperturbed and at constant speeds at the boundaries. All simulations shown were done on a $200 \times 200 \times 400$ grid, with lattice spacing $a=0.2$ and time steps $\Delta t=0.02$, so the Courant condition (here $\Delta t \leq a / \sqrt{3}$ ) holds.

Our simulations are optimized for the deep type-II regime. By solving the two-dimensional, static vortex equations one finds, that in a static straight cosmic string half of the potential energy in the scalar core is contained within a radius $\sqrt{2} m_{\text {scalar }}^{-1} f(\beta)$, where $f$ is a slowly varying function with $f(1)=1, f(64)=1.4$. Lorentzcontraction gives an extra factor $\gamma(v)^{-1}$ in the direction of approach, with $\gamma(v)=1 / \sqrt{1-v^{2}}$. This is the smallest length scale that has to be resolved. We fix its size to match the $\beta=1$ case (without loss of generality we take $\lambda=2, \eta=1$ ). The scalar core is resolved by at least three lattice points up to a center of mass speed of $v \approx 0.94-0.96$, which is indicated explicitly in figure 1 . We define the gauge field core radius to be larger by a factor $\sqrt{\beta}$. The initial string separation is fixed to five times the gauge core radius. For $\beta \leq 64$ the gauge cores still do not overlap in the initial configuration.

\section{Results}

We simulated the collision of cosmic strings at $\beta=1,3.9,4.0,4.1,8,16,31,32,33,49$ and 64 for various speeds $v$ and angles $\alpha$ to find the critical velocity above which the strings effectively pass through each other. The results for $\beta=16$ are shown in figure 1, other $\beta$ values and a detailed discussion can be found in ref. [21].

Our results confirm a downward trend with $\beta$ of the lowest critical velocity $v_{c, m i n}$. Specifically, we find $\left(\beta, v_{c, \min }\right)=(4,0.92),(8,0.92),(16,0.90),(32,0.88)$, $(49,0.88)$ and $(64,0.86)$.

A closer look at the interaction process reveals that the strings do not always intercommute once or twice, as previously observed, but also three and four times for particular values of the initial speed $v$ and angle $\alpha$. An odd number of reconnections results in overall intercommutation of the strings segments, and an even number in the strings effectively passing through, so we can still speak of a critical velocity for the strings passing through. However, each reconnection creates small structure on the strings in the form of a left- and a right-moving kink. In some cases it is not easy to distinguish between one and three, or between two and four reconnections, just by looking at the energy isosurfaces in the intermediate state. But the resulting kinks are clearly visible in the final state and can be counted; in case of doubt we use this criterion (see fig. 3). Successive reconnections therefore lead to left- or right-moving "kink trains", groups of up to four closely spaced kinks (one for each intercommutation). The inter-kink distance within these trains is a few string widths, at formation (see figs 2 and 3 ).

The intercommutation process for type II strings unfolds as follows. After the collision in which the strings exchange ends for the first time two things can happen:

- For $\beta \geq 16$, an expanding loop forms after a short delay. If $v>v_{c}$, the loop catches up with the two original strings and these reconnect again through the loop. This creates a highly curved central region in each string, that move towards each other and can sometimes mediate a third reconnection (see fig. 2). At this point there are two almost anti-aligned string segments and, if they 


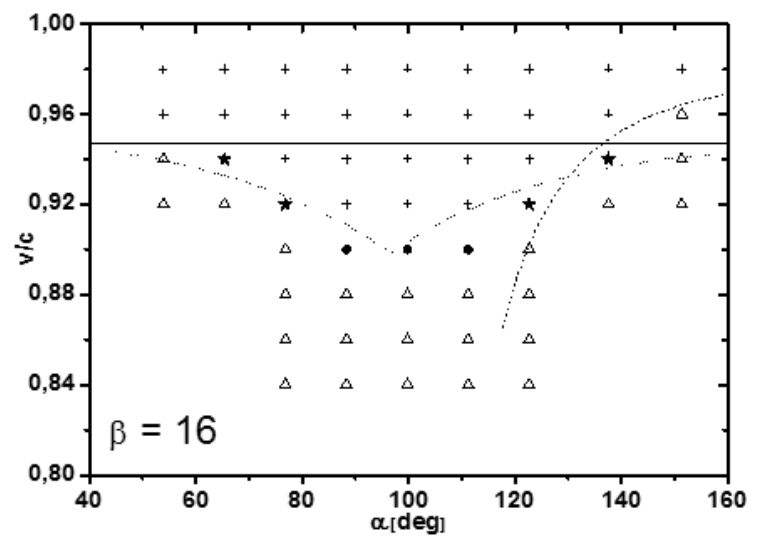

FIG. 1: The number of intercommutations for a range of collision velocities and angles for $\beta=16$. The symbols $\triangle,+, \bullet$ and $\star$ stand for $1,2,3$ or 4 intercommutations respectively. The dotted line is a two-parameter fit based on the Nambu-Goto approximation 13. The dashed line includes core interaction effects at high collision angle $\sim 180^{\circ}$ [21]. Simulations above the horizontal line resolve the scalar core size by less than three lattice points and are therefore less reliable. Triple and quadruple reconnections occur on the boundary betwen single and double reconnections.
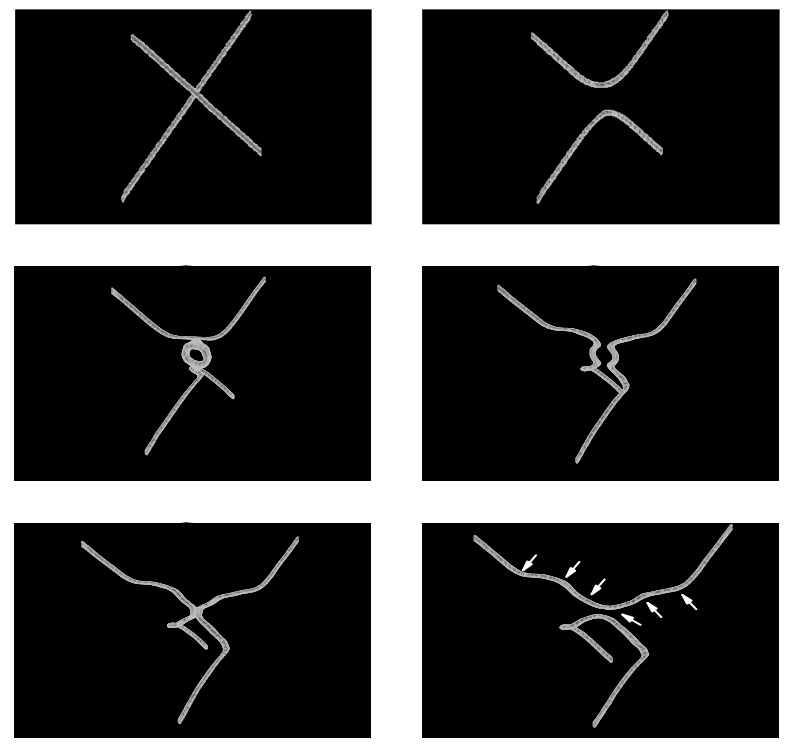

FIG. 2: Scalar field isosurfaces with $|\phi| \leq 0.4$. After the first intercommutation a loop emerges that catches up with the receding strings (second intercommutation), creating two highly curved central regions. These move towards each other and produce a third intercommutation. Two sets of three closely spaced, left- and right-moving kinks are clearly visible on each of the strings (indicated by arrows on the upper string). $(\beta=32, \alpha=122.7, v=0.88), t=3,5,10,17,20,22$

are receding sufficiently slowly, a fourth reconnection is

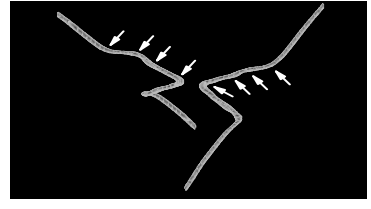

FIG. 3: Typical string configuration after the fourth intercommutation. The arrows show four closely spaced kinks moving up each string; the two corresponding down-moving trains are visible below the collision point. $(\beta=16, \alpha=$ $122.7, v=0.92)$, same isosurfaces, $t=20$.
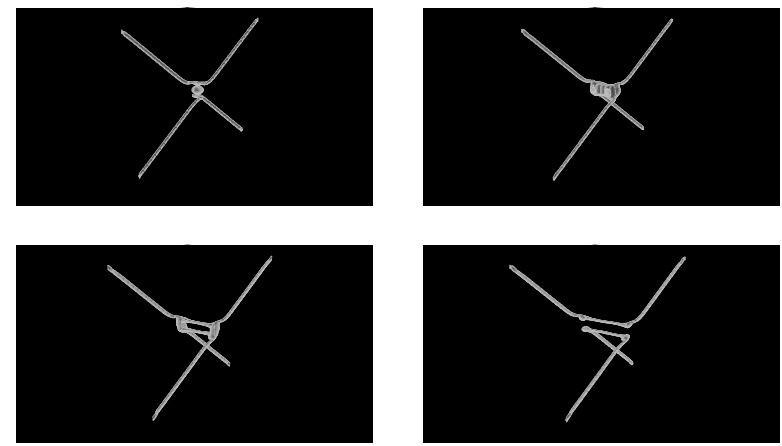

FIG. 4: After the first intercommutation a radiation blob emerges. The blob catches up with the bridge and is absorbed. However, before all radiation is absorbed, a loop seems to be formed. This "loop" is not topological, it breaks resulting in "Dracula's teeth". $(\beta=4, \alpha=122.7, v=0.98)$ $t=2,4.5,6.5,8$.

possible (see fig. 3). This is the largest number of intercommutations we have seen, and only for $\beta=16$ (while triple reconnections are generic). On the other hand, if $v<v_{c}$ the second reconnection does not take place because the string loop does not catch up with the strings. In this case it will contract again and eventually decay into radiation, sometimes after one or a few oscillations.

- For $1<\beta \leq 8$ the energy isosurfaces look somewhat similar, but they reveal a very different intermediate state. The "loop" in figure 4 is just a blob of radiation with no topological features: the (covariant) phase of the Higgs field shows no net winding around the apparent vortex. This is clearly visible in the third timestep, where the "loop" meets the string bridges, breaks and is absorbed - a real vortex loop would not be able to break. In this case the maximum number of reconnections is two.

\section{Discussion}

The interaction of the cores appears to play an important role in interpreting the results. In the type-II regime, parallel strings repel. When strings approach each other, the interaction between the magnetic cores produces a torque that tends to anti-align the string seg- 
ments, so the actual collision angle is higher than in the initial configuration. However, due to this antialignment, the collision results in the annihilation of a larger segment of string so in fact the strings behave as if they that had collided with a smaller angle (which is somewhat counterintuitive and is explained in [21]). For collision angles approaching $180^{\circ}$ this leads to a different prediction for the angular dependence of the critical velocity (fig. 1.)

Our results appear to confirm the claim 13 that the critical speed goes down with increasing $\beta$, from $v_{c} \sim 0.96$ at $\beta=1$ to $v_{c} \sim 0.86$ at $\beta=64$, although this reduction cannot go on indefinitely. A crude (universal) lower bound for $v_{c}$ follows from energy conservation: if the strings anti-align locally and a portion $L$ of each string is annihilated, the maximum energy available to the loop is $2 L \gamma=2 \pi R$, with $R$ the maximum loop radius. If $R<L / 2$ the second intercommutation cannot take place, which happens for $v<\sqrt{1-4 / \pi^{2}} \sim 0.77$. These values of $v_{c}$ are to be contrasted with the average root mean square velocity of the network, which has not yet been investigated for type-II strings (known values range from $v_{r m s}=1 / \sqrt{2} \sim 0.71$ for Nambu-Goto strings in flat space, to $0.63-0.51$ in field theory simulations with $\beta=1$ with cosmic expansion [22, 23]). So, double intercommutations may be less rare than in type-I collisions, but they are still rare events. However, once a kink train is formed its decay time is comparable to that of a single kink, and because of its microscopic size (it is only a few core widths in length) it is very unlikely to be disrupted by intercommutation with another string segment. We conclude that the small scale structure of strongly type-II abelian Higgs string networks could be more clustered than the predictions based on the NambuGoto approximation with $P=1[24-26]$. The effect on cosmological signatures is hard to predict, as multiple intercommutations affect energy loss mechanisms in several, competing, ways. First, if a string self-intersects, an even number of intercommutations frustrates loop formation. Second, kinks tend to suppress the formation of cusps. Third, kink trains would have a stronger gravitational wave signal than single kinks and would give a contribution to the stochastic background not considered previously [27-31]. Finally, the contribution from reconnections is also enhanced in two ways. While we confirm that strongly type-II ANO strings always reconnect $(P=1)$, an effective intercommutation probability $P \leq 1$ due to multiple reconnections will still lead to denser networks and therefore more reconnections. Most of these will be at low velocity, where we expect strong anti-alignment, causing longer segments of string to be annihilated into radiation than for $\beta \approx 1$ strings. So we would expect stronger and more frequent bursts of radiation and cosmic rays than for other string types (lower $\beta$ and also superstrings) where reconnection bursts are always negligible or subdominant [32]. Further work is needed to understand these effects quantitatively, which affect the cosmological bounds. If cosmic strings are ever observed, a crucial question will be whether we can infer their microscopic structure from the observations, in particular whether they are just topological defects or the first evidence of superstring theory in the sky. The results presented here suggest that, even in the relatively simple case of ANO strings, the small scale structure may still hold surprises.

Work supported by the Netherlands Organization for Scientific Research (NWO) under the VICI programme.

* Achucar@lorentz.leidenuniv.nl

† Verbiest@physics.leidenuniv.nl

[1] T. W. B. Kibble, J. Phys. A9, 1387 (1976).

[2] R. Jeannerot, Phys. Rev. D56, 6205 (1997), hep$\mathrm{ph} / 9706391$.

[3] R. Jeannerot, J. Rocher, and M. Sakellariadou, Phys. Rev. D68, 103514 (2003), hep-ph/0308134.

[4] M. Majumdar and A. Christine-Davis, JHEP 03, 056 (2002), hep-th/0202148.

[5] S. Sarangi and S. H. H. Tye, Phys. Lett. B536, 185 (2002), hep-th/0204074.

[6] A. Vilenkin and E. Shellard, Cosmic Strings and Other Topological Defects (Cambridge University Press, 1994).

[7] M. B. Hindmarsh and T. W. B. Kibble, Rept. Prog. Phys. 58, 477 (1995), hep-ph/9411342.

[8] E. J. Copeland and T. W. B. Kibble, Proc. Roy. Soc. Lond. A466, 623 (2010), 0911.1345.

[9] M. Sakellariadou, Nucl. Phys. Proc. Suppl. 192-193, 68 (2009), 0902.0569.

[10] A. Achúcarro and C. J. A. P. Martins, Springer Encyclopaedia of Complexity and Systems Science (2009), 0811.1277.

[11] E. Shellard, Nuc. Phys. B 283, 624 (1987).

[12] R. Matzner, Computers in Physics 2, 51 (1988).

[13] A. Achúcarro and R. de Putter, Phys. Rev. D 74, 121701(R) (2006).

[14] L. M. A. Bettencourt and T. W. B. Kibble, Phys. Lett. B332, 297 (1994), hep-ph/9405221.

[15] E. J. Copeland, T. W. B. Kibble, and D. A. Steer, Phys. Rev. Lett. 97, 021602 (2006), hep-th/0601153.

[16] P. Salmi et al., Phys. Rev. D77, 041701 (2008), 0712.1204

[17] M. G. Jackson, N. T. Jones, and J. Polchinski, JHEP 10, 013 (2005), hep-th/0405229.

[18] A. Avgoustidis and E. P. S. Shellard, Phys. Rev. D73, 041301 (2006), astro-ph/0512582.

[19] A. A. Abrikosov, Sov. Phys. JETP 5, 1174 (1957).

[20] H. B. Nielsen and P. Olesen, Nucl. Phys. B61, 45 (1973).

[21] A. Achúcarro and G. J. Verbiest, in preparation (2010).

[22] J. N. Moore, E. P. S. Shellard, and C. J. A. P. Martins, Phys. Rev. D65, 023503 (2002), hep-ph/0107171.

[23] M. Hindmarsh, S. Stuckey, and N. Bevis, Phys. Rev. D79, 123504 (2009), 0812.1929.

[24] T. W. B. Kibble and E. J. Copeland, Phys. Scripta T36, 153 (1991).

[25] J. Polchinski and J. V. Rocha, Phys. Rev. D74, 083504 (2006), hep-ph/0606205.

[26] E. J. Copeland and T. W. B. Kibble, Phys. Rev. D80, 
123523 (2009), 0909.1960

[27] T. Damour and A. Vilenkin, Phys. Rev. D64, 064008 (2001), gr-qc/0104026.

[28] T. Damour and A. Vilenkin, Phys. Rev. D71, 063510 (2005), hep-th/0410222.

[29] X. Siemens et al., Phys. Rev. D73, 105001 (2006), gr- qc/0603115.

[30] S. Olmez, V. Mandic, and X. Siemens (2010), 1004.0890.

[31] M. Kawasaki, K. Miyamoto, and K. Nakayama (2010), 1002.0652 .

[32] M. G. Jackson and X. Siemens, JHEP 06, 089 (2009), 0901.0867. 